\title{
STATUS OF THOMSON SOURCE AT SPARC/PLASMONX
}

\author{
D. Filippetto, L. Cultrera, G. Di Pirro, M. Ferrario, G. Gatti, C. Vaccarezza, C. Vicario, INFN/LNF, \\ Frascati, Italy \\ A. Bacci, F. Broggi, C. DeMartinis, D. Giove, C. Maroli, V. Petrillo, A. R. Rossi, L. Serafini, \\ P. Tomassini, INFN/MI, Milan, Italy \\ F. Bosi, D. Giulietti, L.A. Gizzi, INFN/CNR-IPCF/Pisa and Univ. of Pisa, Pisa, Italy \\ P. Oliva INFN/Cagliari and Univ. of Sassari, Italy
}

\section{Abstract}

The PLASMONX project foresees the installation at LNF of a $0.25 \mathrm{PW}$ ( $6 \mathrm{~J}, 25$ fs pulse) Ti:Sa laser system FLAME (Frascati Laser for Acceleration and Multidisciplinary Experiments) to operate in close connection with the existent SPARC electron photoinjector, allowing for advanced laser/e-beam interaction experiments. Among the foreseen scientific activities, a Thomson scattering experiment between the SPARC electron bunch and the high power laser will be performed. At the present time the linac has been tested and the electron beam characterized up to the maximum operating energy $(150 \mathrm{MeV})$. The beam lines transporting the beam to the interaction chamber with the laser have been designed.

The electron final focusing system, featuring a quadrupole triplet and large radius solenoid magnet (ensuring an e-beam waist of 5-10 microns) as well as the whole interaction chamber layout have been defined. The optical transfer line issues: transport up to the interaction, tight focusing, diagnostics, fine positioning, have been solved within the final design. The construction of the building hosting the laser has been completed; delivering and installation of the laser, as much of the beam lines elements will take place in the next months.

\section{INTRODUCTION}

The PLASMONX project (PLasma Acceleration and MONochromatic X-ray generation) developed at the National Laboratories in Frascati, close to Rome has entered its last acquisition phase. The project foresees the installation of a Ti:Sa laser system, named FLAME (peak power $>170 \mathrm{TW}$ ), synchronized to the high brightness electron beam produced by the SPARC photo-injector [1]. The electron final focusing system, which will be located in a dedicated beam line, has been defined, as well as the layout of the interaction chamber.

Several experiments are foreseen within this facility, among the others: high gradient plasma acceleration as much as production of mono-chromatic ultra-fast X-ray pulses by Thomson back-scattering (TS), which is hereafter pointed out.

TS X-ray source is attracting strong attention because of its flexibility and potential compactness with respect to conventional synchrotron sources. A TS source driven by high-quality electron beams can work in different operating modes, e.g: the high-flux-moderatemonochromaticity-mode (HFM2) suitable for medical imaging when high-flux sources are needed; the moderate-flux-monochromatic-mode (MFM) suitable to improve the detection/dose performance [2]; short-andmonochromatic-mode (SM) useful for pump-and-probe experiments e.g. in physical-chemistry when tens of femtosecond long monochromatic pulses are needed.

\section{ELECTRON BEAM AND THOMSON INTERACTION REGION}

The electron beam lines design has been completed, with the capability to transport electron beams with energies ranging from $28 \mathrm{MeV}$ up to $150 \mathrm{MeV}$. The key point in electron beam transport is preserving the high brightness coming from the linac, hence ensuring a very tight focusing for the whole energy span. The final features that the electron beam will show at the interaction point, are reported in Tab. 1, while an overall view of the PLASMONX beam lines within the SPARC hall is showed in Fig.1.

Table 1: Electron Beam at the Interaction Point

\begin{tabular}{lc}
\hline \multicolumn{1}{c}{ Parameter } & Value \\
\hline Bunch charge [nC] & $1-2$ \\
Energy [MeV] & $28-150$ \\
Length [ps] & $15-20$ \\
$\varepsilon_{\mathrm{n}, \mathrm{y}}[\mathrm{mm}-\mathrm{mrad}]$ & $1-5$ \\
Energy spread [\%] & $0.05^{*}-0.2$ \\
Spot size at interaction point & $5-10$ \\
rms $[\mu \mathrm{m}]$ & \\
\hline
\end{tabular}

*by using X-band structure

These property of the electron beam are strictly necessary in order to reach the high X Ray beam flux as reported in Tab. $2\left(10^{9}\right.$ photons/sec with a rep rate of 10 $\mathrm{Hz}$ are required to perform the experiment of mammography at SPARC).

Table 2: X-Ray Beam Characteristics (numerically computed).

\begin{tabular}{lc}
\hline \multicolumn{1}{c}{ Parameter } & Value \\
\hline Photon energy [KeV] & $20-500$ \\
Photon numbers (@1nC for & $1.5-2.0 \cdot 10^{9}$ \\
6 mrad collected angle) & \\
\hline
\end{tabular}

The main challenge regarding the electron beam generation is related to the capability to focus down an high charge beam (1-2 nC) to focal spot sizes in the order of 10 microns in the collision point, which in turns implies to accurately take under control emittance and energy spread of the beam itself [3]. 


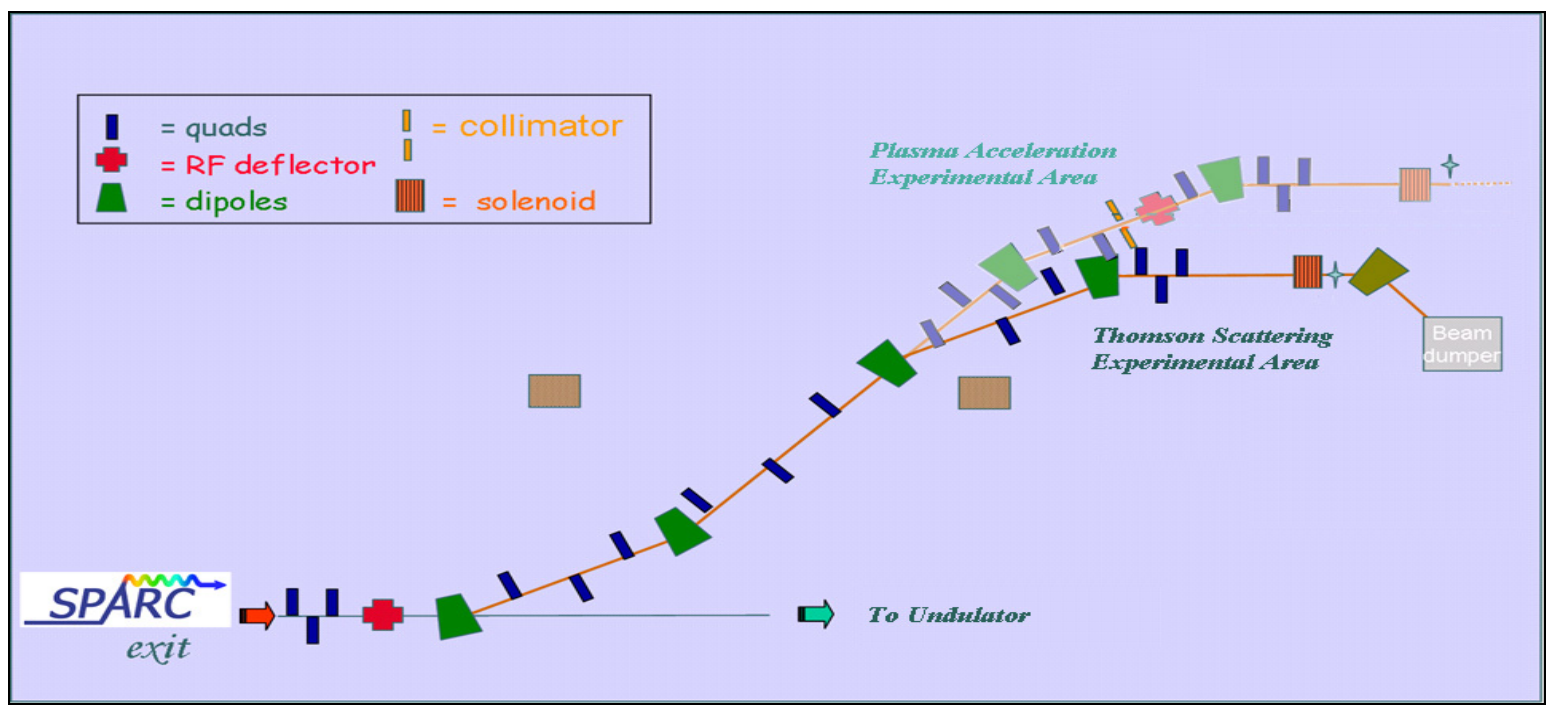

Figure 1: Lay-out of the dog-leg like electron beam line for the TS experimental area.

The emittance growth is controlled by the emittance compensation method, which is one of the main challenges addressed to the SPARC project. The low energy spread values will be obtained by a proper setting of the injection phases into the accelerating structures, which compensates the linear correlation of the longitudinal phase-space, while, in a second step, the use of a X-band short length RF structure [4] will allow to reach a rms energy spread smaller than $5 \cdot 10^{-4}$.

A normal conducting large solenoid has been chosen as the final focusing element. This will ensure a high field on axis $(0.9 \mathrm{~T})$, in spite of the wide aperture in the magnet, necessary for the FLAME laser beam to pass through it avoiding interactions with the internal surface of the vacuum chamber. The project of the solenoid has been finished, and in fig. 2 a sketch of the final engineered model is shown. Also the final dipole meant to dump the electron beam downstream the interaction has been defined; the presence of a high permeability metal shield will prevent the presence of the bipolar field in the interaction point to perturb electrons trajectories. Fig. 3 points out the dipole field profile as modified by the shield, standing to 3D FEM simulations.

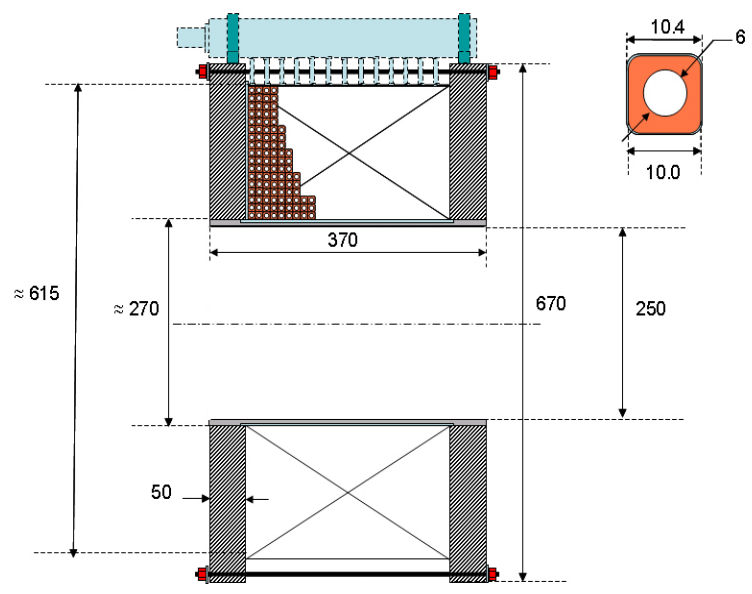

Figure 2: Solenoid engineered scheme.
A preliminary sketch of the interaction chamber has been prepared, in order to fit all the necessary devices (magnetic elements, optical elements, vacuum vessels, diagnostics, etc.) in agreement with the beams transport constraints. The electron beam alignment will be monitored using BPMs and high resolution imaging systems. Time overlapping between laser pulse and electron beam (in the interaction chamber) will be adjusted using an optical delay line, while jitter/delay readout will be made through a picosecond streak camera, by monitoring laser and some kind of electron beam induced radiation (e.g. Cherenkov, transition radiation). More detail will be defined within the next months.

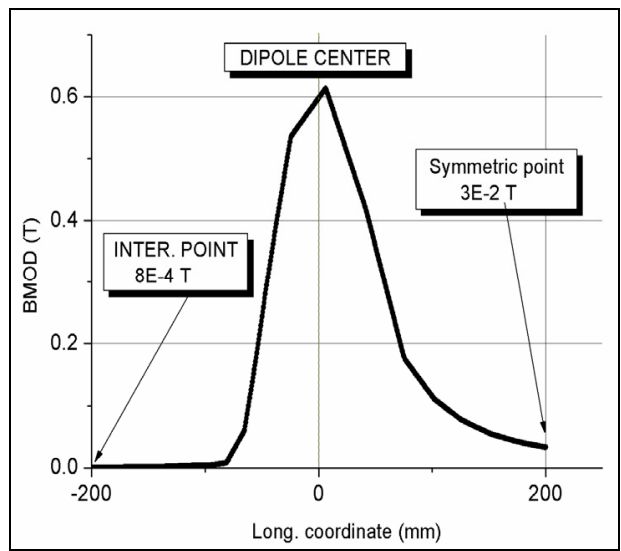

Figure 3:Last dipole field profile.

\section{LASER SYSTEM}

The FLAME laser system is a commercial system from AMPLITUDE technologies [5]. It will deliver more than 200 TW laser pulse to perform Laser-Plasma acceleration and ICS experiments together with the SPARC beam. The laser consists of a femto-source Ti:SA oscillator with a $200 \mathrm{~nm}$ bandwidth and $20 \mathrm{fs}$ pulse duration, pumped by a $4 \mathrm{~W} \mathrm{CW}$ green laser. The oscillator repetition rate is $79.8 \mathrm{MHz}$, corresponding to a 
sub-harmonic of the SPARC RF master clock (RF/36). An active feedback loop assures a laser-to-RF phase lock within 150 fs time jitter. The oscillator pulses are injected into a series of amplification stages working at $10 \mathrm{~Hz}$, up to the level of $650 \mathrm{~mJ}$. The CPA technique is used in order to safely amplify the pulse energy. The pulse is then split to provide simultaneously the beam for the last amplifier and a $50 \mathrm{~mJ}$ probe pulse for the experiments. The last amplifier is based on $5 \mathrm{~cm}$ diameter cryogenic-cooled Ti:SA crystal is excited by ten frequency-doubled Nd:YAG laser that deliver $20 \mathrm{~J}$ total pump energy. The final in-vacuum compressor is used to recompress down the pulse duration at less than 30 fs FWHM for Laser-Plasma acceleration experiments, while in the frame of ICS experiments the required pulse length will be 3-12 ps. In such range the best pulse length - by simulations - has been fixed to be $6 \mathrm{ps}[6]$.

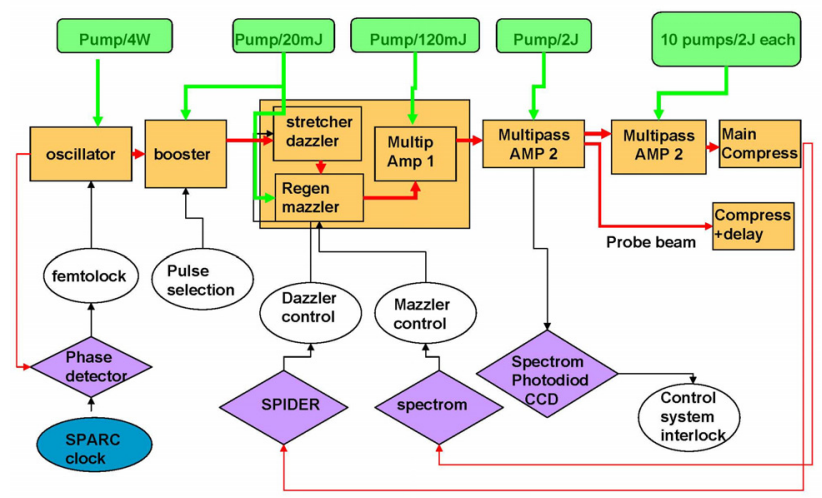

Figure 4: FLAME Laser layout.

In the system design a particular care has been devoted to increase the contrast ratio in order to limit the energy of the fluorescent ns pedestal and the presence of pulses replica. The gain narrowing and the spectral phase distortions are unwanted effects of the CPA that severely limit the minimum pulse duration that can be reached in high energy systems. DAZZLER and MAZZLER [7] are acousto-optic programmable active filters that are included in the system in order to contrast the amplification distortion and minimize the final pulse duration.
After the compressor, the laser beam will be transported to the experimental chambers through 30 meters in vacuum pipe. The beam will be then focussed to the interaction point using an off-axis parabola (focal length $75 \mathrm{~cm}$ and aperture $15 \mathrm{~cm}$ ). A series of diagnostics, controls and motorized optical elements will be useful tools to optimize the overlapping between the laser and the electron beam.

Table 3: List of Expected Laser Beam Parameter

\begin{tabular}{|l|c|}
\hline Wavelength (nm) & 800 \\
\hline Compressed pulse energy (J) & 6 \\
\hline Pulse duration/bandwidth (ps/nm) & $3-12(80)$ \\
\hline Rep. Rate (Hz) & 10 \\
\hline Prepulses contrast & $>10^{\wedge} 6$ \\
\hline Contrast ratio @ 1ns before (ASE) & $>10^{\wedge} 8$ \\
\hline Contrast ratio @ 1-100 ps before & $>10^{\wedge} 6$ \\
\hline Contrat ratio of replica & $>10^{\wedge} 5$ \\
\hline Beam quality $\mathrm{M}^{\wedge} 2$ & $<=1.5$ \\
\hline Energy stability & $10^{\circ} \%$ \\
\hline Pointing stability $(\mu \mathrm{m})$ & $<2$ \\
\hline Synchronization with SPARC clock & $<1 \mathrm{ps}$ \\
\hline
\end{tabular}

Table 3 reports the list of the laser expected parameters. Some performances have already been demonstrated (energy, pulse duration, contrast ratio) at the factory, but they need to be reproduced "in loco". The system will be shipped to Frascati (LNF-INFN) by the summer of 2009.

\section{REFERENCES}

[1] D. Alesini et al., Nucl. Instrum. Methods Phys. Res., Sect. A 507, 345 (2003).

[2] U. Bottigli et al, IL NUOVO CIMENTO, Vol. 29 C, N. 2, Marzo - Aprile 2006

[3] W. J. Brown et al, Phys. Rev. STAB 7 (2004) 060702

[4] D. Alesini et al, Nucl. Instr. and Meth. A 586 (2008) 133-142, doi:10.1016/j.nima.2007.10.040

[5] http://www.amplitude-technologies.com/

[6] P. Tomassini et al, IEEE TRANSACTIONS ON PLASMA SCIENCE, VOL. 36, NO. 4, AUGUST 2008

[7] http://www.fastlite.com 